\title{
Efeitos de práticas corporais no desenvolvimento motor em crianças e adolescentes com paralisia cerebral
}

\author{
Effect of body movement practices on motor development in children and adolescents with cerebral
} palsy

Efecto de las prácticas corporales sobre el desarrollo motor en niños y adolescentes con parálisis cerebral

\author{
Miridan Rodrigues de Oliveira \\ ORCID: https://orcid.org/0000-0001-5861-7423 \\ Centro Universitário Unieuro, Brasil \\ E-mail: miridanoliveira@gmail.com \\ Lucas Evangelista Campos do Nascimento \\ ORCID: https://orcid.org/0000-0002-4233-8582 \\ Centro Universitário Unieuro, Brasil \\ E-mail: lucas.ecampos@outlook.com \\ Bruna Lethicia Jubé Moraes \\ ORCID: https://orcid.org/0000-0002-2307-4956 \\ Centro Universitário Unieuro, Brasil \\ E-mail: brunamoraes301@gmail.com \\ Wesley Salviano de Souza \\ ORCID: https://orcid.org/0000-0003-3960-535X \\ Centro Universitário do Distrito Federal, Brasil \\ E-mail: wesleysalvianoedf@gmail.com \\ Vinícius da Silva Pita \\ ORCID: https://orcid.org/0000-0002-1981-4878 \\ Centro Universitário do Distrito Federal, Brasil \\ E-mail: pita.viniciusedf@gmail.com \\ Geiziane Leite Rodrigues de Melo \\ ORCID: https://orcid.org/0000-0002-7800-2212 \\ Universidade Católica de Brasília, Brasil \\ E-mail: geizianemelo93@gmail.com \\ Rafaello Pinheiro Mazzoccante \\ ORCID: https://orcid.org/0000-0001-9901-8136 \\ Centro Universitário Unieuro, Brasil \\ E-mail: rafa_mazzoccante@hotmail.com
}

\begin{abstract}
Resumo
A paralisia cerebral é oriunda de lesões neurológicas com surgimentos em três diferentes condições, de acordo com a região neuroanatomica lesada, como espástica, atáxica e atetóide. Assim, crianças e adolescentes com paralisia cerebral possuem declínios no controle motor e na realização de movimentos voluntários, o grau de desempenho apresentado será intimamente relacionado a severidade da lesão cerebral. O objetivo desse estudo foi investigar por meio de uma revisão sistemática a influência de diferentes intervenções físicas e motoras no desenvolvimento motor de crianças e adolescentes com PC. Foi realizado uma busca por estudos em inglês e português produzidos de 2010 á 2020, nas bases de dados: Pubmed, Lilacs, Scielo, Elsevier e Pedro. Ao todo foram descritos 06 estudos. Todos os estudos demostram melhoras nas funções investigadas. Os achados expostos nos estudos encontrado nesta revisão, atestam que o efeito de práticas corporais de movimento corrobora de forma positiva no desenvolvimento motor de pacientes com paralisia cerebral. Sugere-se que possam ser realizados mais estudos que aborde não somente os métodos já apresentados, mas novas ferramentas que influencie para a melhora da coordenação motora e da qualidade de vida dos pacientes com paralisia cerebral em todos os seus tipos e topografias.
\end{abstract}

Palavras-chave: Paralisia cerebral; Desenvolvimento motor; Exercício físico.

\section{Abstract}

Cerebral palsy originates from neurological lesions that appear in three different conditions, according to the injured neuroanatomical region, such as spastic, ataxic and athetoid. Thus, children and adolescents with cerebral palsy have declines in motor control and voluntary movements, the degree of performance shown will be closely related to the 
severity of the brain injury. The aim of this study was to investigate through a systematic review the influence of different physical and motor interventions on the motor development of children and adolescents with CP. A search was carried out for studies in English and Portuguese produced from 2010 to 2020, in the databases: Pubmed, Lilacs, Scielo, Elsevier and Pedro. In all, 06 studies were described. All studies show improvements in the investigated functions. The findings exposed in the studies found in this review, demonstrate that the effect of body movement practices corroborates positively on the motor development of patients with cerebral palsy. It is suggested that further studies may be carried out that address not only the methods already presented, but new tools that influence the improvement of motor coordination and quality of life of patients with cerebral palsy in all types and topographies.

Keywords: Cerebral palsy; Motor development; Physical exercise.

\section{Resumen}

La parálisis cerebral se origina por lesiones neurológicas que aparecen en tres condiciones diferentes, según la región neuroanatómica lesionada, como espástica, atáxica y atetoide. Así, los niños y adolescentes con parálisis cerebral tienen disminuciones en el control motor y los movimientos voluntarios, el grado de desempeño mostrado estará íntimamente relacionado con la gravedad de la lesión cerebral. El objetivo de este estudio fue investigar mediante una revisión sistemática la influencia de diferentes intervenciones físicas y motoras en el desarrollo motor de niños y adolescentes con PC. Se realizó una búsqueda de estudios en inglés y portugués producidos de 2010 a 2020 , en las bases de datos: Pubmed, Lilacs, Scielo, Elsevier y Pedro. En total, se describieron 06 estudios. Todos los estudios muestran mejoras en las funciones investigadas. Los hallazgos expuestos en los estudios encontrados en esta revisión, atestiguan que el efecto de las prácticas de movimiento corporal corrobora positivamente en el desarrollo motor de los pacientes con parálisis cerebral. Se sugiere que se puedan realizar más estudios que aborden no solo los métodos ya presentados, sino nuevas herramientas que influyan en la mejora de la coordinación motora y la calidad de vida de los pacientes con parálisis cerebral en todo tipo y topografía.

Palabras clave: Parálisis cerebral; Desarrollo motor; Ejercicio físico.

\section{Introdução}

A paralisia cerebral (PC) apresenta uma prevalência de 2 em 1000 recém-nascidos, esta é definida como uma lesão neurológica de caráter não progressivo, associados ao aparecimento isolado ou conjunto de distúrbios da motricidade e tônus muscular, acometendo diferentes regiões corporais (Dias et al., 2010; Feitoza \& Perez, 2016; Mancini et al., 2016).

As lesões neurológicas causadas pela PC são denominadas em três diferentes classificações, de acordo com a região neuroanatomica lesada, como a espástica caracterizada por disfunção no sistema piramidal, lesão responsável pelo acréscimo desproporcional do tônus muscular e da rigidez muscular, provocando limitações de movimento (Corrêa et al., 2012; Piovezani et al., 2017). Na condição classificada como atáxica, há lesões oriundas das regiões cerebelares, condições resultante na diminuição da força muscular, incoordenação motora e tremores (Corrêa et al., 2012; Piovezani et al., 2017). Por fim a atetòide, caracteriza-se por alteração no sistema extrapiramidal, alterações resultantes em dissinergia do movimento, instabilidade no tônus muscular e comprometimento na coordenação motora (Corrêa et al., 2012; Piovezani et al., 2017).

Diante disso há três tipos da PC que podem exercer declínios no controle motor e dificuldades na execução de movimentos voluntários em crianças e adolescentes(Corrêa et al., 2012; Grecco et al., 2013; Kara et al., 2019; Rosa et al., 2008; Strapasson, 2009). Todavia, a condição de mobilidade, controle motor e movimento será inteiramente dependente do grau de severidade da lesão causada pela PC (Grecco et al., 2013; Kara et al., 2019; Rosa et al., 2008; Strapasson, 2009)

Mediante a esse cenário, pesquisas recentes veem demonstrando os benefícios da inserção de práticas corporais e de exercício físico como, o treinamento de força, treinamento de macha na esteira, equoterapia e atividades aquáticas foram benéficas em diminuir os prejuízos atrelados a função motora grossa e no equilíbrio dinâmico e estático de crianças e adolescentes com PC (Grecco et al., 2013; Kara et al., 2019; Rosa et al., 2008; Strapasson, 2009).

Akinola et al. (2019), verificaram o efeito de 10 semanas de intervenção através de exercícios aquáticos em crianças com PC, após realização foi evidenciado aumento nas funções motoras grossas, como deitar, rolar, sentar, engatinhar, ajoelhar e ficar de pé. Achado semelhante foi observado no estudo de Correa et al (2012), onde constataram melhoras no equilíbrio do voluntario, após 10 sessões de equoterapia em criança com PC. 
Pesquisas recentes com público infantil e adolescente demonstram amostras e métodos bem heterogêneos, torna-se necessário reunir os principais resultados voltados a estudos do comportamento motor em crianças e adolescentes com paralisia cerebral, para tal assertiva auxiliar os profissionais atuantes na prescrição de exercícios físicos a este público. Por outro lado, auxiliar os pesquisadores na perspectiva inicial dos principais achados de estudos anteriores para orientar a produção de estudos futuros com o olhar destinado a intervenção do exercício físico com o público do estudo (Sousa et al., 2012).

Deste modo, o presente estudo tem como objetivo verificar por meio de uma revisão sistemática as diferentes intervenções físicas e motoras no desenvolvimento motor de crianças e adolescentes com paralisia cerebral.

\section{Metodologia}

Trata-se de um estudo transversal, com análise descritiva e abordagem de seleção de artigos com metodologias quantitativa (Pereira et al., 2018) . A busca foi realizada em periódicos indexados nas bases de dados eletrônicas SCIELO, LILACS, PUBMED, ELSEVER e PEDRO, sobre o efeito de intervenções físicas e motoras no desenvolvimento motor de crianças e adolescentes com paralisia cerebral. No período de setembro de 2010 a setembro de 2020, usando os seguintes descritores: Paralisia Cerebral (Cerebral Palsy), Desenvolvimento motor (Motor Development), Exercício Físico (Physical Exercise), Motricidade (Motricity), Tônus Muscular (Muscle Tone), Técnicas de Fisioterapia (Physiotherapy Techniques), Crianças (Childern), Adolescentes (Teenager), combinados com operador boleano AND tanto na língua inglesa e portuguesa.

Os critérios de inclusão foram: artigos originais, estudos publicados entre 2010 a 2020, amostra com crianças e adolescentes com paralisia cerebral e exercício físico. Foram excluídos estudos que realizados com adultos, estudos descritivos, estudos repetidos e estudos que não avaliava o desenvolvimento motor. A avaliação os estudos seguiu as etapas conforme Whittemore e Knafl (2005).

\section{Resultados e Discussão}

As informações sobre as etapas do desenvolvimento de busca e escolha dos artigos validados para esta revisão estão na Figura 1. As informações contidas nos trabalhos estão presentes na Tabela 1. 
Figura 1. Fluxograma que contém as etapas do processo de busca e seleção dos artigos incluídos na presente revisão. A partir das palavras-chave foram identificados, inicialmente, 168 artigos potenciais sendo que as combinações foram: Cerebral Palsy AND motor AND Motor AND physical exercise OR Child OR Teenager AND Motricity. Na etapa triagem, 85 artigos que foram selecionados por meio da leitura dos títulos, resumo e conclusões. Todavia, 24 estudos foram elegíveis conforme os critérios de exclusão. A partir disso, 6 artigos foram considerados elegíveis para o estudo, sendo 5 ensaios clínicos randomizados e 01 sendo estudo de caso.

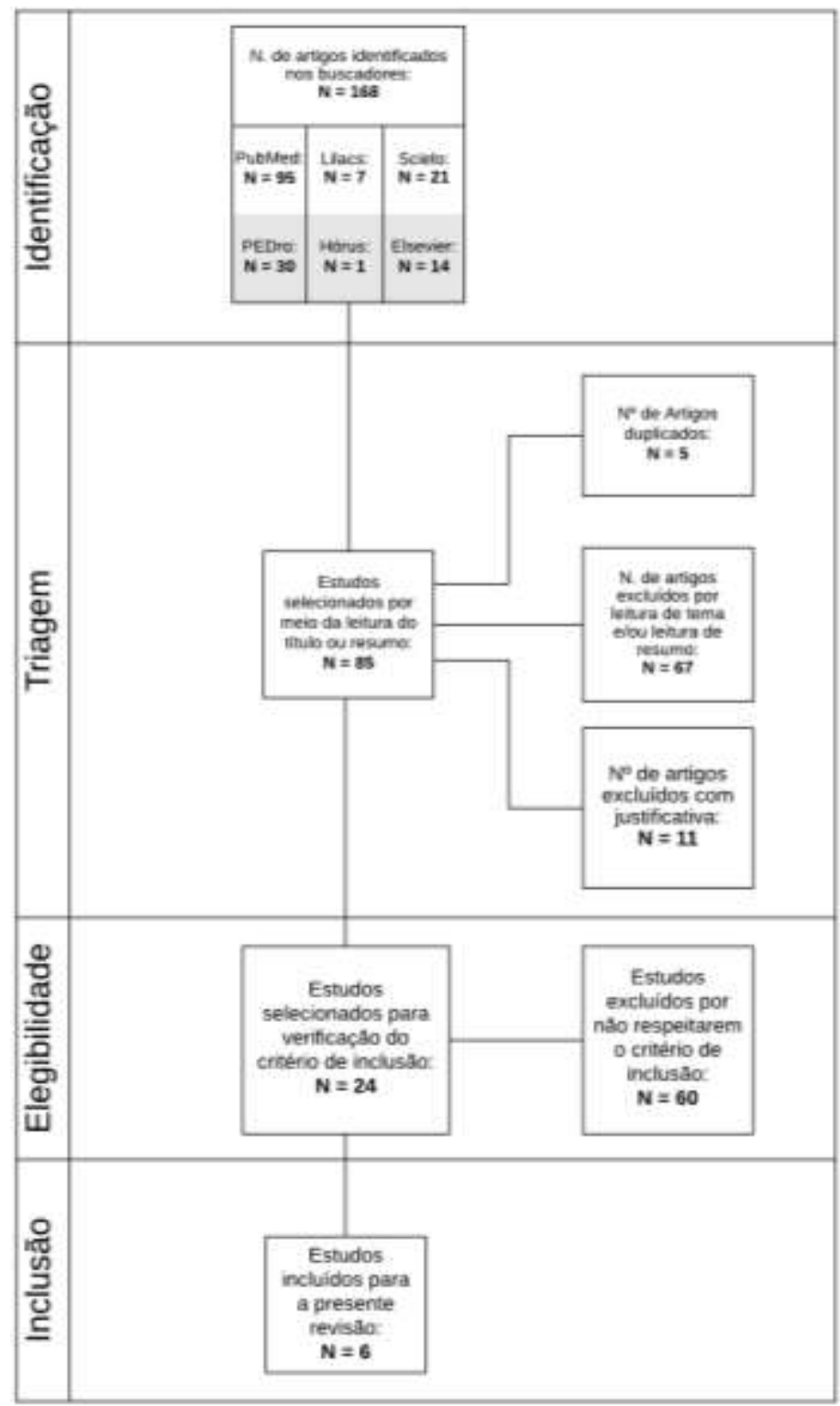

Fonte: Autores.

Os métodos, amostra, tipo de estudo, protocolo de intervenção, resultados e identificação dos estudos selecionados durante esta revisão, estão descritos na Tabela 1. Observa-se que a faixa etária variou entre 1 a 16 anos de idade, com amostras entre 1 a 33 indivíduos, totalizando 120 participantes. A amostra foi composta pelos três tipos de paralisia cerebral, sendo 03 estudos com tipo espástica, 01 atetóide e 02 que não foram identificadas nos estudos, e por fim 02 estudos com predominância topográfica quadriplégica. Nos estudos selecionados se observou que (90\%) da amostra era do sexo masculino. Além disso, os estudos escolhidos foram realizados entre os anos de 2012 até 2019, em que (50\%) dos mesmos foram desenvolvidos no Brasil, os outros (50\%) divididos entre, 01 na Nigéria, 01 Sérvia e 01 na Turquia. 


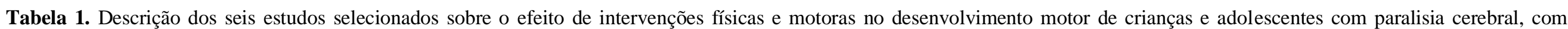
cronologia de 2012 até 2019.

\begin{tabular}{|c|c|c|c|c|c|}
\hline Nome/Ano & Tipo de Estudo & Amostra & Métodos & Protocolo de intervenção & Resultados \\
\hline $\begin{array}{l}\text { Akinola et al. } \\
\text { (2019) }\end{array}$ & Ensaio Clínico & $\begin{array}{l}30 \text { crianças de } 01 \text { a } 12 \\
\text { anos com PC Espástica }\end{array}$ & $\begin{array}{l}\text { As crianças foram divididas em dois grupos GE e } \\
\text { GC. Avaliaram o nível de mobilidade através do } \\
\text { Gross motor function classification system } \\
\text { (GMFCS) e a função motora grossa } \\
\text { foi definida por meio do Gross } \\
\text { motor function measur - } 88 .\end{array}$ & $\begin{array}{l}\text { Ambos os grupos participaram de } 20 \\
\text { sessões por } 10 \text { semanas consecutivas } \\
\text { de } 2 \text { encontros semanais. O GE } \\
\text { realizou treinamento físico na água, } \\
\text { alongamento passivo manual e } \\
\text { treinamento funcional, enquanto o GC } \\
\text { realizou os mesmos exercícios em } \\
\text { solo. }\end{array}$ & $\begin{array}{c}\text { Melhora na função motora } \\
\text { grossa. }\end{array}$ \\
\hline $\begin{array}{l}\text { Moraes et al. } \\
\qquad(2016)\end{array}$ & Ensaio Clínico & $\begin{array}{l}15 \text { crianças com Paralisia } \\
\text { Cerebral com idade de } 05 \\
\text { a } 10 \text { anos. }\end{array}$ & $\begin{array}{l}\text { A plataforma de força AMTI ACCUSWAY Plus } \\
\text { (ADVANCE MECHANICAL TECHONOLOGIES, INC), } \\
\text { foi utilizada para medir o equilíbrio postural. A } \\
\text { topografia } \\
\text { (hemipartesia, diparesia e quadriparesia) e a } \\
\text { GROSS MOTOR FUNCTION CLASSIFICATION } \\
\text { SYSTEM (GMFCS) foram usados para caracterizar } \\
\text { Os participantes. O Equilibrio Dinâmico foi } \\
\text { avaliado pelo BBS e foi utilizado a parte I e II do } \\
\text { PEDI para medir desempenho funcional. }\end{array}$ & $\begin{array}{l}\text { O programa de equoterapia foi } \\
\text { realizado por } 30 \text { min, } 02 \text { vezes } \\
\text { semanais em um período de } 12 \\
\text { semanas. Foram utilizados } 03 \text { cavalos } \\
\text { treinados e equipados com manta, } \\
\text { cabo e estribo. }\end{array}$ & $\begin{array}{l}\text { Foram observados melhora no } \\
\text { equilíbrio postural e dinâmico } \\
\text { e nas tarefas motoras. }\end{array}$ \\
\hline $\begin{array}{l}\text { Dimitrijevic et al. } \\
\qquad \text { (2012) }\end{array}$ & Ensaio Clínico & $\begin{array}{l}27 \text { crianças com } \\
\text { PC espástica constituídas } \\
\text { por } 04 \text { hemiplégicas, } 06 \\
\text { diplegicas, } 13 \text { tetraplégicas } \\
\text { e } 04 \text { hemiplégicas, com } \\
\text { idade entre } 05 \text { e } 14 \text { anos }\end{array}$ & $\begin{array}{l}\text { A GMFM- } 88 \text { foi utilizado para mensurar a função } \\
\text { motora grossa da amostra, e quanto o teste ALYN } \\
2 \text { (WOTA 2) avaliou o nível de ajuste e função do } \\
\text { nadador. As medidas descritivas } \\
\text { e características do tipo de PC e o nível expandido } \\
\text { e revisado da escala de classificação da função } \\
\text { motora grossa (GMFCS) }\end{array}$ & $\begin{array}{l}\text { Durante } 06 \text { semanas foram } \\
\text { ministradas sessões de } 55 \text { minutos e } \\
02 \text { encontros semanais. O primeiro } 10 \\
\text { min foi realizado aquecimento leve na } \\
\text { água, } 40 \text { min de técnica de natação e } \\
05 \text { min finais de jogos. }\end{array}$ & $\begin{array}{l}\text { Melhora significativa na função } \\
\text { motora grossa e nas } \\
\text { habilidades aquáticas. }\end{array}$ \\
\hline
\end{tabular}


Grecco et al. Ensaio Clínico (2013) Randomizado Controlado entre 03 e 12 anos, com níveis I, II e III do sistema de classificação função motora grossa (GMFCS), e de pressão TEKSCAN MaTSCaN SYSTEM (BOSTON, que estivesse em deambulação funcional por pelo menos 12 meses.

Avaliação do equilíbrio funcional foi medido por meio da escala BERG, enquanto o equilíbrio estático foi mensurado através da

sessões semanais de 30 min durante de macha em esteira) realiza a velocidade de $60 \%$ nos primeiros e (esteira) quanto estável (solo) ultimos 5 minutos $80 \%$ nos 20 min 0 trazem melhoras no equilíbrio GC (treinamento de macha terrestre), funcional e estático. EUA), as crianças realizaram o

velocidade de treniun ATL para determinar a Entretanto, em esteira confortável durante os primeiros 5 min resultou em uma melhora e últimos, e 20 min de aumentava a maior no equilíbrio funcional e velocidade da macha. menor oscilação médio-lateral.

Kara et al. (2019) Ensaio

33 participantes com idade GROSS MOTOR FUNCTION CLASSIFICATION entre 07 e 16 anos, SYSTEM EXPANDED AND REVISED (GMFCS-E e R) classificados como nível I foram usados para classificar a função motora pelo GMFCS. Divididos em grossa. As habilidades manuais foram medidas 2 grupos, 17 no GE (treinamento de força progressiva) e 16 no GC (comparação ativa).
ESTADIÔMETRO e BALANÇA para o pes composição corporal foi avaliada pelo IMC.
3 sessões semanais durante $60 \mathrm{~min}$, durante 12 semanas, o GRUPO

funcional combinado com

CONTROLE realizou sessões que não exercícios pliométricos melhor incluía treinamento de força de ou a macha, a função motora resistência progressiva. Já o GRUPO grossa, o equilíbrio dinâmico, EXPERIMENTAL realizou 3 sessões por força muscular e a potência. semana durante $90 \mathrm{~min}$, por 12 semanas. No qual o protocolo era constituído por fortalecimento funcional usando o leg press, exercício pliometrico e treinamento de equilíbrio.
Corrêa et al. (2012)
Estudo de Caso

Uma menina de 05 anos

com paralisia

cerebral atetóide.
Foi utilizado o teste de equilíbrio de BERG (BERG BALANCE SCALE) e a intervenção terapêutica se deu por sessões de EQUOTERAPIA.
A coleta de dados foi realizada pré e pós teste pela escala de equilíbrio de BERG. Foram realizadas 10 sessões de equoterapia, com duração de $30 \mathrm{~min}$ cada e com intervalo de 07 dias entre elas. Nas sessões utilizaram diferentes variações de andaduras do cavalo (passo), de piso (areia/gramado) e mudança de direção e de combinação de movimento.
O estudo concluí que a equoterapia parece ter contribuído na melhora do equilíbrio, no tratamento de paralisia atetóide.

*PC- PARALISIA CEREBRAL / IMC- INDICE DE MASSA CORPORAL / *GE- GRUPO EXPERIMENTAL / *GC- GRUPO CONTROLE Fonte: Autores. 
$\mathrm{Na}$ Tabela 1 se observa que para mensurar a função motora grossa foi utilizado o Gross Motor Function Classification System (GMFCS) estando presente em (80\%) dos artigos apresentavam níveis de classificação I, II e III, os quais são classificados de acordo com a mobilidade de cada nível, em que I se refere a paticipantes que se deslocam sem limitações; II traz consigo participantes que necessitam de auxílio para se locomover e III faz uso de dispositivos manuais como bengalas para se deslocar (Palisano et al., 2007). Além disso, foi utilizado para avaliar o equilíbrio a plataforma de força Amti Accusway Pluz, a escala de equilíbrio Berg e a Estabilometria.

Os protocolos de intervenções utilizados nos estudos foram (20\%) atividades aquáticas, destes (10\%) foram treinamento de força no meio aquático e (10\%) exercícios de técnica de natação. Dois utilizaram a intervenção por meio da equoterapia, um o treinamento de força com exercícios pliométricos associados ao treinamento de macha em esteira.

\section{Discussão}

Os achados contidos nesta revisão sistemática, demonstram que independentemente da amostra encontrada nos estudos (faixa etária e acometimento da PC), todas as pesquisas apresentadas atestam avanços nas funções investigadas, como a melhora na coordenação motora grossa, no equilíbrio dinâmico e estático (Akinola et al., 2019; Corrêa et al., 2012; Dimitrijević et al., 2012; Grecco et al., 2013; Moraes et al., 2018).

Akinola et al. (2019) comprovam que exercícios no meio aquático, durante 10 semanas, em crianças com PC espástica, foi capaz de promover melhora na coordenação motora grossa no grupo intervenção, quando comparado ao grupo controle. Em concordância, Dimitrijevic et al (2012) averiguaram o efeito de 6 semanas de exercícios de habilidades aquáticas, baseados nas técnicas da modalidade da natação, após período de realização notou-se respostas benéficas na melhora da coordenação motora dos participantes da intervenção. Apesar da intervenção utilizada nos estudos serem semelhantes, os métodos aplicados, e duração das mediações foram diferentes, todavia ambos obtiveram resultados positivos em relação a coordenação motora.

Ademais, outras formas de exercício físico se mostram benéficas as pessoas com PC, em sua pesquisa Grecco et al (2013) certificam que o treinamento de marcha, tanto em esteira quanto no solo, durante 7 semanas, foram capazes de melhorar o equilíbrio estático e dinâmico no grupo intervenção, em comparação ao grupo controle, propiciando até a diminuição da oscilação médio-lateral na macha. Achados similares foram encontrados por Kara et al (2019) que verificaram os efeitos do treinamento de potência em crianças com PC hemiplégica, após 12 semanas de prática, os resultados demonstraram melhora no equilíbrio estático e dinâmico (Timed Up and Go) nos colaboradores submetidos ao treinamento de força.

Em contrapartida, Moraes et al (2018) realizou um treinamento de 12 semanas, por meio da equoterapia, percebeu melhoras no equilíbrio postural, equilíbrio dinâmico e nas tarefas motoras dos participantes submetidos ao exercício. Resultado que corrobora com pesquisa feita por Correa et al (2012) que após aplicação de 10 sessões de equoterapia, em criança com PC atetóide, obteve melhorias significativas no equilíbrio do participante.

Nota-se a importância de práticas terapêuticas por meio do exercício físico em suas diferentes aplicações, como ferramenta benéfica em progresso da motricidade de crianças e adolescentes com paralisia cerebral. Tal afirmativa relacionase a um avanço positivo, tanto nos aspectos físicos, fisiológicos, psicológicos e cognitivos dessa população (Dimitrijević et al., 2012; Sousa et al., 2012).

Portanto o presente estudo demonstra real relevância em sua construção para profissionais da saúde e pessoas com paralisia cerebral, em salientar os inúmeros efeitos benéficos do exercício físico, como auxilio, reduzindo limitações musculares e motoras advindos da paralisia cerebral, e também destacando a profissionais da área da saúde atuantes, a 
importância de intervenções motrizes e sobre os efeitos de práticas diversificadas de movimentos e seus respectivos benefícios em particular.

Em outra perspectiva esta revisão vem a orientar novos pesquisadores em referência aos estudos presentes na área de intervenção motora e crianças e adolescentes com paralisia cerebral, tal descritiva pode assim nortear a elaboração de novos estudos na área com o objetivo de elucidar ainda mais os efeitos do exercício físico neste público.

\section{Conclusão}

Os achados expostos nos estudos utilizados nesta revisão, demostram que o efeito de práticas corporais favorece de forma positiva no desenvolvimento motor de pacientes com paralisia cerebral. Sugere-se que possam ser realizados mais estudos que abordem não somente os métodos já apresentados, mas novas ferramentas que apresente resultados positivos na melhoria da coordenação motora e da qualidade de vida dos pacientes com paralisia cerebral em todos os seus tipos e topografias. Os programas de exercício físico em diferentes protocolos mostram-se interessantes em auxiliar pessoas com paralisia cerebral em aumento e/ou manutenção motriz e tônus muscular, mas, é interessantes novas propostas investigando os efeitos de diferentes protocolos de intervenção em beneficiar estas ações para auxiliar e orientar os profissionais atuantes em programas de promoção e reabilitação motriz e muscular com este público.

\section{Referências}

Akinola, B. I., Gbiri, C. A., \& Odebiyi, D. O. (2019). Effect of a 10-Week Aquatic Exercise Training Program on Gross Motor Function in Children With Spastic Cerebral Palsy. Global Pediatric Health, 6, 2333794X1985737. https://doi.org/10.1177/2333794x19857378

Corrêa, R. G., Tonon, É., \& Suter, T. M. da C. (2012). A Influência Da Equoterapia No Equíbrio De Paciente Com Paralisia Cerebral. Revista Horus, 7(3), 18. http://periodicos.estacio.br/index.php/revistahorus/article/viewFile/4024/1851

Dias, A. C. B., Freitas, J. C., Formiga, C. K. M. R., \& Viana, F. P. (2010). Desempenho funcional de crianças com paralisia cerebral participantes de tratamento multidisciplinar. Fisioterapia e Pesquisa, 17(3), 225-229. https://doi.org/10.1590/s1809-29502010000300007

Dimitrijević, L., Aleksandrović, M., Madić, D., Okičić, T., Radovanović, D., \& Daly, D. (2012). The effect of aquatic intervention on the gross motor function and aquatic skills in children with cerebral palsy. Journal of Human Kinetics, 32(1), 167-174. https://doi.org/10.2478/v10078-012-0033-5

Feitoza, F. B., \& Perez, F. da S. B. (2016). Efeitos Da Intervenção Fisioterapêutica Na Reabilitaçâo De Pacientes Com Paralisia Cerebral Discinética Distônica. http://www.faculdadealfredonasser.edu.br/files/Pesquisar_5/21-11-2016-21.34.28.pdf

Grecco, L. A. C., Tomita, S. M., Christovão, T. C. L., Pasini, H., Sampaio, L. M. M., \& Oliveira, C. S. (2013). Effect of tre admill gait training on static and functional balance in children with cerebral palsy: A randomized controlled trial. Brazilian Journal of Physical Therapy, 17(1), 17-23. https://doi.org/10.1590/S1413-35552012005000066

Kara, O. K., Livanelioglu, A., Yardimci, B. N., \& Soylu, A. R. (2019). The Effects of Functional Progressive Strength and Power Training in Children With Unilateral Cerebral Palsy. Pediatric Physical Therapy, 31(3), 286-295. https://doi.org/10.1097/PEP.0000000000000628

Mancini, M., Schaper, C., Figueiredo, E., Sampaio, R., \& Coelho, Z. (2016). The effects of hippotherapy on postural balance and functional ability in chidren with cerebral palsy. Journal of Physical Therapy Science, 25(8), 2220-2226. https://doi.org/10.1589/jpts28.2220

Moraes, A. G., Copetti, F., Angelo, V. R., Chiavoloni, L. L., \& Ana Cristina David. (2018). The Effects of Hippotherapy on the Oral Motor and Respiratory Systems in Children with Cerebral Palsy. Multiple Sclerosis Journal, 24(10), 2220-2226.

Palisano, R., Rosenbaum, P., Bartlett, D., Livingston, M., Walter, S., Russell, D., Wood, E., Galuppi, B., \& , G., Brasileira Traduzido por Daniela Baleroni Rodrigues Silva, V., \& Iara Pfeifer Carolina Araújo Rodrigues Funayama, L. (2007). GMFCS-E \& R- Sistema de Classificação da Função Motora Grossa. Reference: Dev Med Child Neurol, 39, 214-223. www.canchild.ca

Pereira, A. S., Shitsuka, Dorlivete Moreira Parreira, F. J., \& Shitsuka, R. (2018). Metodologia da Pesquisa Científica - Licenciatura em Computação. https://repositorio.ufsm.br/bitstream/handle/1/15824/Lic_Computacao_Metodologia-Pesquisa-Cientifica.pdf?sequence=1.

Piovezani, J. C., Maitschuk, M. M., Oliva, F. S., Brandalize, D., \& Brandalize, M. (2017). Método Pediasuit melhora a função motora grossa de criança com paralisia cerebral atáxica. ConScientiae Saúde, 16(1), 131-138. https://doi.org/10.5585/conssaude.v16n1.6689 
Research, Society and Development, v. 10, n. 2, e31710212627, 2021

(CC BY 4.0) | ISSN 2525-3409 | DOI: http://dx.doi.org/10.33448/rsd-v10i2.12627

Rosa, G. K. B., Marques, I., Medina-Papst, J., \& Gobbi, L. T. B. (2008). Desenvolvimento motor de criança com paralisia cerebral: avaliação e intervenção. Revista Brasileira de Educação Especial, 14(2), 163-176. https://doi.org/10.1590/s1413-65382008000200002

Sousa, A. A. De, Teixeira-arroyo, C., \& Paulista, U. E. (2012). Benefícios do exercício físico na paralisia cerebral : uma revisão crítica. Fafibe On-Line, 5, 7. Strapasson, A. M. (2009). "Polybat": um jogo para pessoas com paralisia cerebral. "Polybat": Um Jogo Para Pessoas Com Paralisia Cerebral, 23(2), 121133. https://doi.org/10.1590/S1807-55092009000200003

Whittemore, R., \& Knafl, K. (2005). The integrative review: updated methodology. JAN Leading Global Nursing Research, 52(5), 546-553. https://doi.org/https://doi.org/10.1111/j.1365-2648.2005.03621.x 